\title{
Analysis of risk factors of non-suicidal self-harm behavior in adolescents with depression
}

\author{
Can Shao, Xiaomeng Wang, Qingyan Ma, Yunzhi Zhao, Xiaobin Yun \\ Department of Psychiatry, the First Affiliated Hospital of Xi'an Jiaotong University, Xi'an, China \\ Contributions: (I) Conception and design: C Shao; (II) Administrative support: C Shao; (III) Provision of study materials or patients: X Wang, C Shao; \\ (IV) Collection and assembly of data: All authors; (V) Data analysis and interpretation: C Shao; (VI) Manuscript writing: All authors; (VII) Final \\ approval of manuscript: All authors. \\ Correspondence to: Can Shao. Department of Psychiatry, the First Affiliated Hospital of Xi'an Jiaotong University, 277 West Yanta Road, Xi'an 710061, \\ China. Email: shao1234561030@163.com.
}

Background Adolescents are a high-risk group for non-suicidal self-injury (NSSI), which seriously affects
their physical and mental health. This study aimed to explore the risk factors for depressive adolescents with
NSSI.
Methods: A total of 153 adolescents with depression were divided into the NSSI group (n=65) and non-
NSSI group (n=88) according to the criteria stipulated by Diagnostic and Statistical Manual of Mental
Disorders, 5th Edition (DSM-5). The Beck scale for suicidal ideation (BSS), adolescent self-rating life
events checklist (ASLEC), family adaptability and cohesion evaluation scale II-Chinese version (FACES II-
CV), childhood trauma questionnaire short form (CTQ SF), and multidimensional scale of perceived social
support (MSPSS) were applied to evaluate suicidal ideation, frequency and intensity of stressful life events,
family functions, childhood trauma, and perceived support, respectively. We applied two-dimensional logistic
regression to identify risk factors for NSSI.

Results: Female gender ratio, suicidal ideation, and attempted suicide were significantly higher in the NSSI group than in the non-NSSI group (all $\mathrm{P}<0.05$ ). Scores of interpersonal relationships in ASLEC, emotional abuse, and emotional neglect in the CTQ-SF were significantly higher in the NSSI group than those in the non-NSSI group (all $\mathrm{P}<0.001)$. The scores of family cohesion $(\mathrm{P}=0.001)$ and family adaptability $(\mathrm{P}=0.01)$ were significantly lower in the NSSI group than in the non-NSSI group. The MSPSS was used to assess support from the family, and the index was significantly lower in the NSSI group $(\mathrm{P}<0.001)$. After adjusting for age and gender, BSS score, interpersonal relationship score, emotional abuse score, and emotional neglect score were identified as independent risk factors for NSSI.

Conclusions: The rate of NSSI in adolescents with depression is high. Higher scores of BSS, interpersonal relationship, emotional abuse, and neglect were independently associated with NSSI.

Keywords: Adolescents; depression; non-suicidal self-injuries (NSSIs); risk factor

Submitted Jun 29, 2021. Accepted for publication Aug 11, 2021.

doi: 10.21037/apm-21-1951

View this article at: https://dx.doi.org/10.21037/apm-21-1951

\section{Introduction}

Non-suicidal self-injury (NSSI) refers to the behavior of deliberately, repeatedly, and systematically injuring one's own tissues and organs without the purpose of ending one's life (1). The traditional view is that NSSI is closely related to borderline personality disorder. However, the latest research has shown that NSSI is also common in two-way affective disorder, post-traumatic stress disorder, and anxiety disorder (2). Although most NSSI patients have no definite intention of suicide, they often overlap, and NSSI behavior increases the risk of suicidal ideation or behavior by over 20 
times throughout their lifetime (3). Because NSSI behavior can release pressure or negative emotions and then induce a positive sensory experience, NSSI-implementing individuals seldom take the initiative to seek medical treatment. Adolescents often enact NSSI, and patients with depression have a greater risk of NSSI implementation than those without depression (4). A meta-analysis also showed that NSSI was closely related to depression (5). The etiology and mechanism of NSSI are complex. Previous studies have suggested that female gender, transgender experience, low self-esteem, and interpersonal relationships are risk factors for NSSI in transgender people (6). Current studies suggest that NSSI is correlated with childhood trauma, family dysfunction, and individual emotional instability. Therefore, early identification and intervention of NSSI risk factors in adolescent depression patients have essential and positive clinical and social significance. In this study, we intended to explore the risk factors of NSSI in adolescent depression patients to provide a theoretical and reference basis for the future prevention of NSSI.

We present the following article in accordance with the STROBE reporting checklist (available at https://dx.doi. org/10.21037/apm-21-1951).

\section{Methods}

\section{Participants}

From October 2019 to February 2021, adolescent depression patients in our hospital were selected as research participants. The inclusion criteria were as follows: (I) patients aged 12-18 years old; (II) the patients met the diagnostic criteria of depression in Diagnostic and Statistical Manual of Mental Disorders, 5th Edition (DSM-5); (III) when the patients were included in the study, their condition was stable, mood was stable, and they were able to complete the relevant scale tests; (IV) the patients provided informed consent to the study. The exclusion criteria were as follows: (I) patients with other mental disorders, such as schizophrenia, bipolar disorder, drug abuse, and so on; (II) patients with severe organic diseases, such as severe liver and kidney dysfunction; (III) patients who refused to participate in this study. According to the DSM-5 criteria for NSSI, the participants were divided into the NSSI group and the non-NSSI group. All procedures performed in this study involving human participants were in accordance with the Declaration of Helsinki (as revised in 2013). The study was approved by the Ethics Committee of the First Affiliated Hospital of Xi'an Jiaotong University (No.: KYLLSL-2019-244) and informed consent was taken from all the patients.

\section{Research methods}

\section{General participant information}

The general information of participants, such as age, gender, education level, family income, and course of the disease, was collected using Excel software (Microsoft, Redmond, WA, USA).

\section{Self-rating anxiety scale (SAS) and self-rating depression scale (SDS)}

The SAS and SDS were composed of 20 items, and each item was scored with four grades (1-4 points) to evaluate the severity of anxiety and depression in the first week. The corresponding standard score was obtained by multiplying the total score of SAS and SDS items by 1.25. The SDS standard scores of normal, mild, moderate, and severe depression were $<53,53-62,63-72$, and $>72$, respectively. According to the national norm, the SAS standard scores of normal, mild, moderate, and severe anxiety were $<50$, $50-59,60-69$, and $\geq 70$, respectively.

\section{Beck scale for suicidal ideation (BSS)}

The BSS scale mainly evaluates the level of suicidal ideation of patients. The scale was completed in two steps: if the answers to questions 4 and 5 in the first five were "no", the participant was considered to have no suicidal ideation, and the evaluation ended. If the answer to question 4 or 5 was "weak", "medium", or "strong", the participant was required to complete the following questions and the total score of questions 6-19 was calculated.

\section{Adolescent self-rating life events checklist (ASLEC)} The ASLEC scale consists of 27 items, with 1-5 points for each item, covering six factors: interpersonal relationship, learning pressure, punishment, loss, health adaptation, and others. It can be used to evaluate the frequency and intensity of stressful life events in adolescents.

\section{Family adaptability and cohesion evaluation scale (FACES II-CV)}

The FACES II-CV scale has 30 items in total. It mainly evaluates the emotional degree between family members (family intimacy) and the ability of a family unit to make 
Table 1 Comparison of general data of NSSI patients and non-NSSI patients

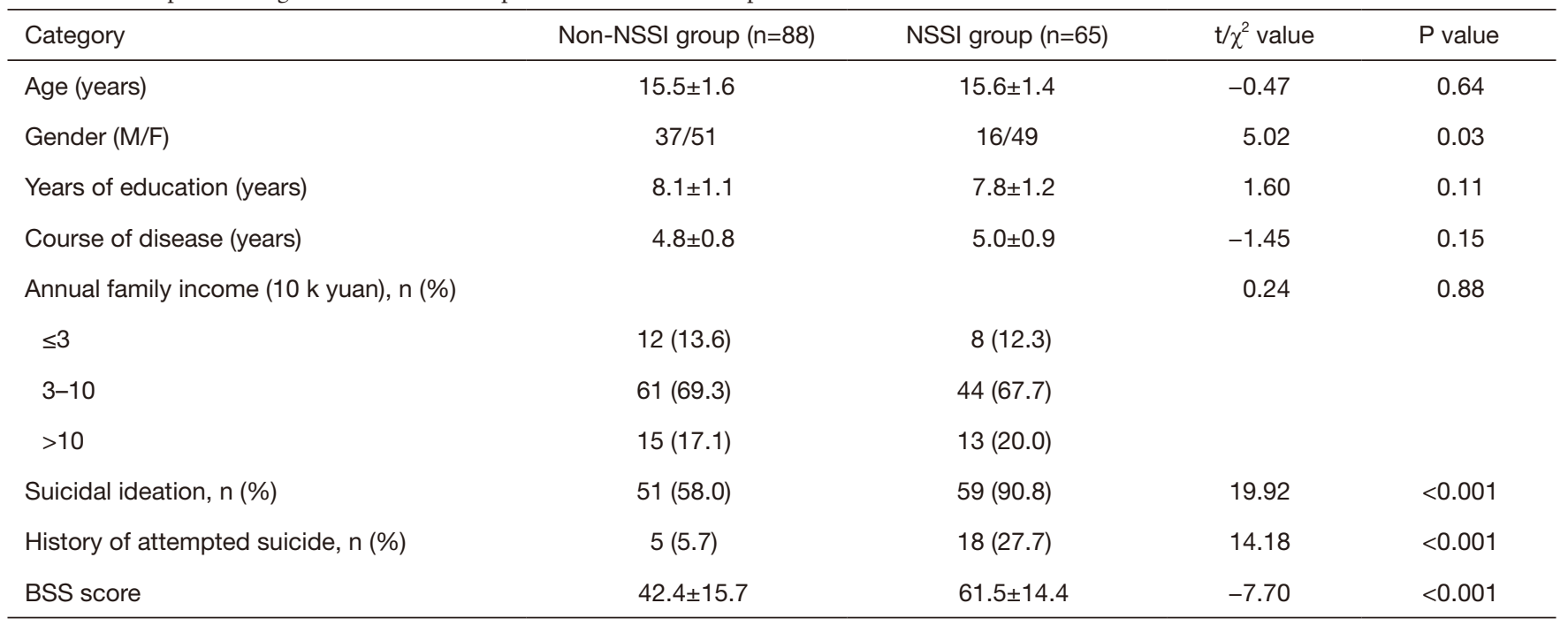

NSSI, non-suicidal self-injury; BSS, Beck scale for suicidal ideation.

corresponding changes (family adaptability) to new problems in the family situation and stages of development.

\section{Childhood trauma questionnaire-short form (CTQ-SF)}

The CTQ-SF is one of the most commonly used tools to measure childhood abuse and neglect. There are 28 items in the questionnaire. Each item has 1-5 points, including five dimensions: physical abuse, sexual abuse, emotional abuse, physical neglect, and emotional neglect.

\section{Multidimensional scale of perceived social support (MSPSS)}

The MSPSS scale includes 12 items, which mainly assess the degree of support patients feel from family, friends, and others.

\section{Statistical analysis}

The software SPSS 22.0 (IBM Corp., Chicago, IL, USA) was used for statistical analysis, and GraphPad Prism 8.0 (GraphPad Software, LA Jolla, CA, USA) was used for image production. Chi-square test, Fisher's exact probability method, or independent Student's $t$-test were used to compare the general information and the rating scales between the NSSI group and non-NSSI group. Using with NSSI and without NSSI as dependent variables, binary logistic regression was used to analyze the independent risk factors of NSSI. Bilateral $\mathrm{P}<0.05$ indicated a statistically significant difference.

\section{Results}

\section{General participant information}

A total of 153 patients with adolescent depression were included in the analysis, including 88 patients without NSSI and 65 with NSSI. There was no significant difference in average age, years of education, course of disease, and annual family income between the two groups (all $\mathrm{P}>0.05$ ). The proportion of women, suicide ideation, and suicide attempt history in patients with NSSI were significantly higher than those in patients without NSSI (all $\mathrm{P}<0.05$ ), as shown in Table 1.

\section{Comparison of SAS, SDS, ASLEC, and FACES II-CV between the two groups}

As shown in Table 2, there was no significant difference in SAS and SDS scores between the two groups (all $\mathrm{P}>0.05)$. The ASLEC scale showed that the interpersonal relationship score of patients with NSSI was significantly higher than that of patients without NSSI $(\mathrm{P}<0.001)$. The FACES II-CV scale revealed that family cohesion $(\mathrm{P}=0.001)$ and family adaptability $(\mathrm{P}=0.01)$ of patients with NSSI were significantly lower than those of participants without NSSI.

\section{Comparison of CTQ-SF and MSPSS between the two groups}

As shown in Table 3, the CTQ-SF scores of emotional abuse 
Table 2 Comparison of SAS, SDS, ASLEC, and FACES II-CV between patients with and without NSSI

\begin{tabular}{|c|c|c|c|c|}
\hline Category & Non-NSSI group $(\mathrm{n}=88)$ & NSSI group (n=65) & t-value & $P$ value \\
\hline SDS score & $67.1 \pm 12.7$ & $71.2 \pm 14.2$ & -1.88 & 0.06 \\
\hline \multicolumn{5}{|l|}{ ASLEC scale } \\
\hline Interpersonal relationship & $10.5 \pm 2.0$ & $16.0 \pm 4.5$ & -9.21 & $<0.001$ \\
\hline Punishment & $11.2 \pm 7.0$ & $11.9 \pm 8.4$ & -0.56 & 0.58 \\
\hline Losing & $4.8 \pm 2.4$ & $4.5 \pm 2.1$ & 0.82 & 0.41 \\
\hline Health adaptation & $6.8 \pm 2.8$ & $7.8 \pm 3.6$ & -1.55 & 0.12 \\
\hline Others & $6.8 \pm 3.2$ & $7.2 \pm 4.4$ & -0.65 & 0.52 \\
\hline Family adaptability & $33.2 \pm 11.7$ & $28.5 \pm 10.5$ & 2.56 & 0.01 \\
\hline
\end{tabular}

SAS, self-rating anxiety scale; SDS, self-rating depression scale; ASLEC, adolescent self-rating life events checklist; FACES II-CV, family adaptability and cohesion evaluation scale II-Chinese version; NSSI, non-suicidal self-injury.

and neglect in participants with NSSI were significantly higher than those in participants without NSSI (all $\mathrm{P}<0.001)$. The MSPSS scale showed that the support from family members of NSSI participants was significantly lower than that of those without NSSI $(\mathrm{P}<0.001)$.

\section{Multi factor analysis of NSSI}

As shown in Table 4 and Figure 1, after adjusting for age and gender, BSS score, interpersonal relationship, emotional abuse, and emotional neglect were independent risk factors for NSSI.

\section{Discussion}

Studies have shown that depression has a relatively low incidence rate in children but that it significantly increases in adolescence, and is related to specific biological and sociological factors (7). Although there are effective treatment schemes for depression, the diagnosis of adolescent depression is often missed. Therefore, early identification, diagnosis, and intervention are essential to improve the quality of life and prognosis of adolescent depression patients. At present, cognitive behavior therapy is the main treatment mode of NSSI, which specifically includes syndrome differentiation behavior therapy and mood regulation group therapy. The behavior of NSSI is unique, a cause of concern for parents and clinicians, and an independent predictor of suicide. Therefore, thorough investigation of NSSI is of great clinical significance.

In this study, the detection rate of NSSI was $42.5 \%$, similar to the $45.45 \%$ reported by other teams in China. We found that adolescent depression patients with NSSI are mainly female, which was consistent with most previous reports (8); however, some studies have shown that there is no significant relationship between NSSI behavior and female gender.

The BSS score of NSSI participants was significantly higher than that of those without NSSI, suggesting that NSSI may be closely related to suicidal ideation or behavior. At present, whether there is a strict distinction between NSSI and attempted suicide remains controversial. Some studies have asserted that the risk of suicidal ideation or suicidal behavior is significantly higher in NSSI patients, both of which belong to the same disease spectrum, while other researchers believe that the function of NSSI is a form of releasing pressure, and its purpose is to avoid suicide (9).

Compared with the non-NSSI group, the interpersonal function of the NSSI group was significantly decreased, and family cohesion and adaptability were impaired. Some studies have suggested that interpersonal conflict may be one of the factors that promote NSSI (10). According to 
Table 3 Comparison of CTQ-SF MSPSS score between patients with and without NSSI

\begin{tabular}{|c|c|c|c|c|}
\hline Category & Non-NSSI group $(n=88)$ & NSSI group $(n=65)$ & $\mathrm{t}$-value & $P$ value \\
\hline Physical abuse & $7.8 \pm 4.2$ & $8.2 \pm 4.4$ & -0.57 & 0.57 \\
\hline Sexual abuse & $6.1 \pm 3.9$ & $6.2 \pm 3.2$ & -0.17 & 0.86 \\
\hline Emotional abuse & $10.5 \pm 3.2$ & $15.3 \pm 4.1$ & -7.84 & $<0.001$ \\
\hline Emotional neglect & $13.8 \pm 5.8$ & $18.2 \pm 6.3$ & -4.47 & $<0.001$ \\
\hline \multicolumn{5}{|l|}{ MSPSS scale } \\
\hline Family & $23.1 \pm 6.4$ & $16.9 \pm 4.9$ & -6.79 & $<0.001$ \\
\hline Friends & $17.8 \pm 6.7$ & $18.2 \pm 7.0$ & -0.36 & 0.72 \\
\hline
\end{tabular}

CTQ-SF, childhood trauma questionnaire-short form; MSPSS, multidimensional scale of perceived social support; NSSI, non-suicidal selfinjury.

Table 4 Binary logistic regression analysis results of NSSI

\begin{tabular}{lcccc}
\hline Regression factor & B & Exp(B) & $95 \%$ Cl & 0.10 \\
\hline Age & -1.38 & 0.25 & $0.05-1.29$ & $0.33-8.75$ \\
Gender & 1.68 & 5.38 & $1.07-2.32$ & $0.02^{*}$ \\
BSS score & 0.46 & 1.58 & $1.40-17.54$ & $0.02^{*}$ \\
Interpersonal relationship & 1.98 & 7.26 & $0.92-1.22$ & 0.47 \\
Family intimacy & 0.05 & 1.05 & $0.77-1.01$ & 0.08 \\
Family adaptability & -0.13 & 0.88 & $1.05-13.02$ & $0.04^{*}$ \\
Emotional abuse & 1.31 & 3.70 & $1.06-1.84$ & $0.03^{*}$ \\
Emotional neglect & 0.29 & 1.34 & $0.43-1.00$ & 0.05 \\
MSPSS-family & -0.43 & 0.65 & 0
\end{tabular}

*, $\mathrm{P}<0.05$. NSSI, non-suicidal self-injury; Cl, confidence interval; BSS, Beck scale for suicidal ideation; MSPSS, multidimensional scale of perceived social support.

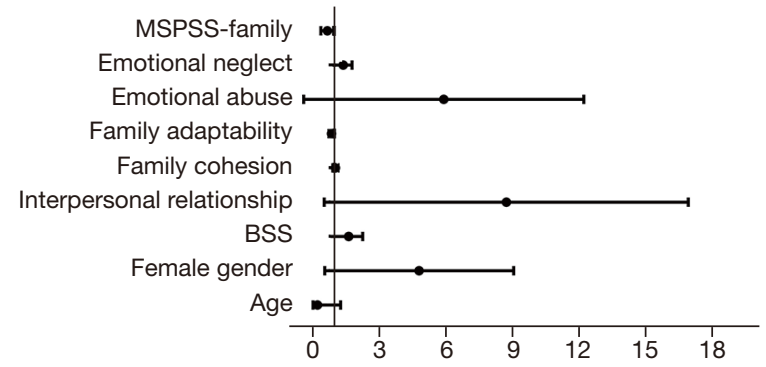

Figure 1 Binary logistic regression analysis forest chart of NSSI. NSSI, non-suicidal self-injury; MSPSS, multidimensional scale of perceived social support; BSS, Beck scale for suicidal ideation. the theory of the intentional self-injury emotion avoidance model, affective factors (such as interpersonal conflict) lead to strong adverse effects, combine with the defective emotional management mechanism, and then lead to NSSI (11). Normally functioning families can provide physical space and psychological comfort for growing adolescents. Family conflict, disharmony among family members, and decreased family adaptability are closely related to NSSI. For example, Nemati et al. found that low family function increases the risk of NSSI in adolescents (12). Cassels et al. confirmed that low family function is 
associated with NSSI in adolescents (13).

This study found that childhood trauma, especially emotional abuse and emotional neglect, are independent risk factors for NSSI in adolescent depression. Emotional abuse refers to verbal attacks, humiliation, and demeaning of others. In contrast, emotional neglect refers to the neglect or denial of personal emotional needs by parents or others for an extended duration. Brown et al. discussed the relationship between emotional abuse, emotional neglect, and NSSI among adolescents. It was found that $72 \%$ of NSSI patients had been subjected to emotional abuse. The path analysis model suggested that emotional neglect played a mediating effect in NSSI (14). Glassman et al. found that the correlation between emotional abuse in childhood and NSSI in adolescents was the most significant (15).

The results of MSPSS suggested that the perceived family support score of participants with NSSI was lower than that of those without NSSI, meaning that family and social support may have a particular impact on the development of NSSI. Previous studies have shown that depressed patients with family function or family environment defects lack support when encountering stressful events, which has a certain impact on the suicidal behavior of patients (16). Long-term follow-up observation found that family support had the most far-reaching influence on when NSSI was initiated, maintained, and stopped (17).

Binary logistic regression analysis showed that only BSS score, interpersonal relationship, emotional abuse, and emotional support were independent risk factors after NSSI was adjusted for age and gender. We speculate that this may be related to the interaction between different scales. For example, studies have found that family function is associated with BSS suicidal ideation in patients with mental disorders (18).

The limitations of this study include that it was a crosssectional study, so it is impossible to assess the causal relationship between independent predictors and NSSI behavior. In addition, the frequency and intensity of NSSI were not considered in this study, and it was not clear whether the independent predictors were related to the severity of NSSI.

In conclusion, this study found that the prevalence of NSSI among adolescent depression patients was high. The scores of BSS, interpersonal relationship, emotional abuse, and emotional neglect were the independent risk factors of NSSI, which has provided a theoretical basis for early intervention.

\section{Acknowledgments}

Funding: None.

\section{Footnote}

Reporting Checklist: The authors have completed the STROBE reporting checklist. Available at https://dx.doi. org/10.21037/apm-21-1951

Data Sharing Statement: Available at https://dx.doi. org/10.21037/apm-21-1951

Conflicts of Interest: All authors have completed the ICMJE uniform disclosure form (available at https://dx.doi. org/10.21037/apm-21-1951). The authors have no conflicts of interest to declare.

Ethical Statement: The authors are accountable for all aspects of the work in ensuring that questions related to the accuracy or integrity of any part of the work are appropriately investigated and resolved. All procedures performed in this study involving human participants were in accordance with the Declaration of Helsinki (as revised in 2013). The study was approved by the Ethics Committee of the First Affiliated Hospital of Xi'an Jiaotong University (No.: KYLLSL-2019-244) and informed consent was taken from all the patients.

Open Access Statement: This is an Open Access article distributed in accordance with the Creative Commons Attribution-NonCommercial-NoDerivs 4.0 International License (CC BY-NC-ND 4.0), which permits the noncommercial replication and distribution of the article with the strict proviso that no changes or edits are made and the original work is properly cited (including links to both the formal publication through the relevant DOI and the license). See: https://creativecommons.org/licenses/by-nc-nd/4.0/.

\section{References}

1. Nock MK. Why do people hurt themselves? New insights into the nature and functions of self-injury. Curr Dir Psychol Sci 2009;18:78-83.

2. Bentley KH, Cassiello-Robbins CF, Vittorio L, et al. The association between nonsuicidal self-injury and the emotional disorders: a meta-analytic review. Clin Psychol Rev 2015;37:72-88. 
3. Whitlock J, Muehlenkamp J, Eckenrode J, et al. Nonsuicidal self-injury as a gateway to suicide in young adults. J Adolesc Health 2013;52:486-92.

4. Jacobson CM, Hill RM, Pettit JW, et al. The association of interpersonal and intrapersonal emotional experiences with non-suicidal self-injury in young adults. Arch Suicide Res 2015;19:401-13.

5. Fox KR, Franklin JC, Ribeiro JD, et al. Meta-analysis of risk factors for nonsuicidal self-injury. Clin Psychol Rev 2015;42:156-67.

6. Arcelus J, Claes L, Witcomb GL, et al. Risk factors for non-suicidal self-injury among trans youth. J Sex Med 2016;13:402-12.

7. Cyranowski JM, Frank E, Young E, et al. Adolescent onset of the gender difference in lifetime rates of major depression: a theoretical model. Arch Gen Psychiatry 2000;57:21-7.

8. Bresin K, Schoenleber M. Gender differences in the prevalence of nonsuicidal self-injury: A meta-analysis. Clin Psychol Rev 2015;38:55-64.

9. Grandclerc S, De Labrouhe D, Spodenkiewicz M, et al. Relations between nonsuicidal self-injury and suicidal behavior in adolescence: a systematic review. PLoS One 2016;11:e0153760.

10. Guerry JD, Prinstein MJ. Longitudinal prediction of adolescent nonsuicidal self-injury: examination of a cognitive vulnerability-stress model. J Clin Child Adolesc Psychol 2010;39:77-89.

11. Chapman AL, Gratz KL, Brown MZ. Solving the puzzle of deliberate self-harm: the experiential avoidance model.

Cite this article as: Shao C, Wang X, Ma Q, Zhao Y, Yun X. Analysis of risk factors of non-suicidal self-harm behavior in adolescents with depression. Ann Palliat Med 2021;10(9):96079613. doi: 10.21037/apm-21-1951
Behav Res Ther 2006;44:371-94.

12. Nemati H, Sahebihagh MH, Mahmoodi M, et al. Nonsuicidal self-injury and its relationship with family psychological function and perceived social support among Iranian high school students. J Res Health Sci 2020;20:e0469.

13. Cassels M, van Harmelen AL, Neufeld S, et al. Poor family functioning mediates the link between childhood adversity and adolescent nonsuicidal self-injury. J Child Psychol Psychiatry 2018;59:881-7.

14. Brown RC, Heines S, Witt A, et al. The impact of child maltreatment on non-suicidal self-injury: data from a representative sample of the general population. BMC Psychiatry 2018;18:181.

15. Glassman LH, Weierich MR, Hooley JM, et al. Child maltreatment, non-suicidal self-injury, and the mediating role of self-criticism. Behav Res Ther 2007;45:2483-90.

16. Tompson MC, Langer DA, Asarnow JR. Development and efficacy of a family-focused treatment for depression in childhood. J Affect Disord 2020;276:686-95.

17. Tatnell R, Kelada L, Hasking P, et al. Longitudinal analysis of adolescent NSSI: the role of intrapersonal and interpersonal factors. J Abnorm Child Psychol 2014;42:885-96.

18. Lipschitz JM, Yen S, Weinstock LM, et al. Adolescent and caregiver perception of family functioning: relation to suicide ideation and attempts. Psychiatry Res 2012;200:400-3.

(English Language Editor: J. Jones) 